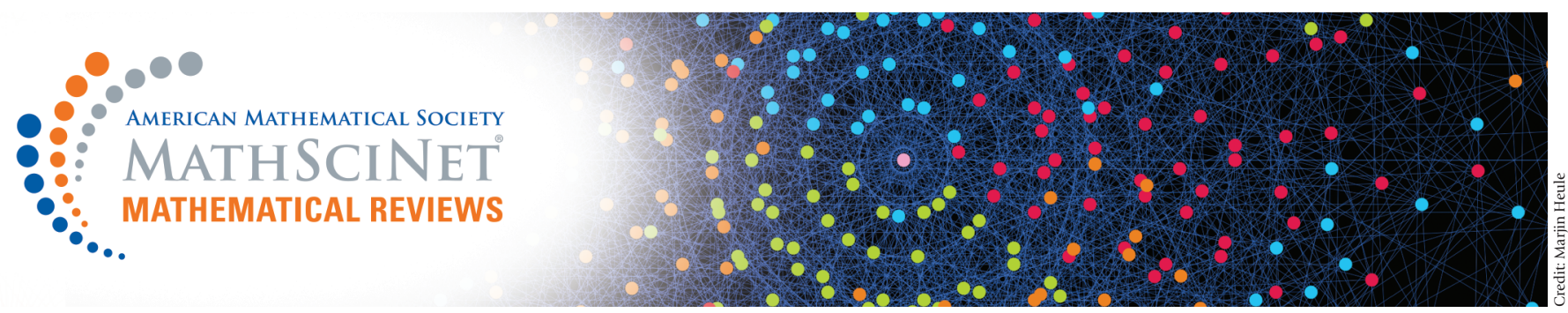

\title{
Don't Count on It
}

\section{Edward Dunne}

There is a well-known dictum in business: "If you can't measure it, you can't manage it." You may see it on its own or as part of SMART Goals (Specific, Measurable, Achievable, Realistic, and Timely). The dictum is very tempting. For one thing, numbers are seen as being neutral, free from bias. In a narrow sense, this is true. In the broader sense, we know that the algorithms and programs used to produce those numbers are often not free from bias. It is also tempting because CEOs, managers, and consultants envision applying all sorts of tools from engineering and the sciences to optimizing the measured quantities. (I look forward to the day that a management consultant's report includes Euler-Lagrange equations.) There is, however, a corollary: "You get what you measure." This is not meant to be affirming. It is, rather, a refutation. The corollary, in the context of a school administration that evaluates faculty based on students' scores on standardized tests, explains why a teacher will "teach to the test." By using student evaluations to assess teaching faculty, many colleges and universities inadvertently encourage grade inflation since positive evaluations correlate with higher course grades. CEOs will focus intently on stock prices, instead of product quality or employee relations, because that is the measure their bosses-the shareholders-apply to them. Since the metrics drive the rewards systems, the results of the stimulus-response system are not surprising. If the metric is the

Edward Dunne is the executive editor of Mathematical Reviews at the American Mathematical Society. His email address is egd@ams.org.

For permission to reprint this article, please contact: reprint-permi ssion aams.org.

DOI: https://dx.doi.org/10.1090/noti2201 message, people will learn to game the system. In academia, the measured quantities are known as "bibliometrics."

The best-known bibliometrics are Impact Factors for journals and citation counts for authors. I had originally planned to discuss both types, but chose to focus on citation counts for space considerations, leaving journal bibliometrics for another time. Citation counts are available from multiple sources, including Clarivate's Web of Science, Google Scholar, and MathSciNet. When people refer to "Highly Cited Researchers" (HCRs), though, they generally mean the list created by Clarivate, which is freely available [https://recognition.webofsciencegroup . com/awards/highly-cited/2019/]. It is interesting to consider the list of 89 mathematicians who are Highly Cited Researchers.

\section{Citation Counts and Influential Researchers}

Using the Mathematical Reviews database, which is what powers MathSciNet, one can identify the primary subject area of each HCR, as determined by the Mathematics Subject Classification (MSC) in which the majority of their papers were published. The first thing to notice is that this group is unrepresentative of mathematics in general. For instance, there are no algebraists or geometers, and only one number theorist, in the group. Of the 10 largest subject classes in the MR database (counting journal articles published from 2008 to 2018), only six are represented as the primary subjects of the HCRs. Overall, only 16 of a possible 60 subject areas are represented. If we also look at secondary areas of the researchers (defined as an area in which the researcher has published at least half as many papers as in the primary area), we reach 21 of the possible 60 subject areas. The number of HCRs whose primary subject is the 
given area are listed under the header "Count." The number of HCRs whose primary or secondary subject is the given area are listed under "Count.+ ." There is a noticeable skewing towards analysis and certain applications, as can be seen in Table 1 . Looking only at the HCRs' primary subjects, those subjects represent $45 \%$ of the articles published in journals from 2008 to 2018 (inclusive). Looking at the HCRs' primary and secondary subjects, they represent $52 \%$ of the articles published in journals from 2008 to 2018 (inclusive). The following chart compares the distribution of subjects of the HCRs with the distribution in the full Mathematical Reviews Database (MRDB). Note: The full MSC has 63 classes, but I am considering just 60 of them, as three subject areas are unlikely to show up as among HCRs or among the prize winners discussed below: $00=$ General, $01=$ History and biography, 97=Mathematics education.

Here is another way to look at the HCRs in mathematics. According to the Web of Science Group at Clarivate Analytics, the list recognizes "the world's most influential researchers of the past decade." The determination is made based on citation counts. An alternative list of influential researchers in mathematics may be created by combining the lists of winners of the AMS prizes, the SIAM prizes, the EMS prizes, and the IMU prizes, including the Fields Medal. There are 636 such winners of the 29 such prizes, over all time for the prizes. As of this writing, only five of the 89 HCRs in mathematics have won any of the prizes (Franco Brezzi, Luis Caffarelli, Emmanuel Candès, Stanley Osher, and Terry Tao). Rather than comparing all 636 prize winners, I looked instead at the 103 mathematicians who had won at least two of the prizes. This gives a comparable number of researchers as in the HCR list. The primary research areas of these prize winners represent 27 of the 60 classes. Including primary and secondary research areas, the prize winners represent 36 of the classes. The subjects represented are more diverse and, in particular, they are not concentrated in analysis and applications. The two most frequently occurring subjects are algebraic geometry and number theory, which hardly show up among the HCRs. Looking at the prize winners' primary subjects, those subjects represent $61 \%$ of the articles published in journals from 2008 to 2018 (inclusive). Looking at the prize winners' primary and secondary subjects, they represent $76 \%$ of the articles published in journals from 2008 to 2018 (inclusive).

\begin{tabular}{|c|c|c|c|c|c|}
\hline \multicolumn{6}{|c|}{ Subject areas for HCRs } \\
\hline MSC & Subject & $\begin{array}{l}\text { Rank in } \\
\text { MRDB }\end{array}$ & $\begin{array}{l}\text { Count } \\
\text { in HCR }\end{array}$ & $\begin{array}{l}\% \text { in } \\
\text { HCR }\end{array}$ & $\begin{array}{l}\text { Count+ } \\
\text { in HCR }\end{array}$ \\
\hline 35 & Partial differential equations & 1 & 20 & $22.50 \%$ & 23 \\
\hline 62 & Statistics & 3 & 14 & $15.70 \%$ & 14 \\
\hline 34 & $\begin{array}{l}\text { Ordinary differential } \\
\text { equations }\end{array}$ & 11 & 13 & $14.60 \%$ & 17 \\
\hline 65 & Numerical analysis & 2 & 13 & $14.60 \%$ & 16 \\
\hline 47 & Operator theory & 16 & 9 & $10.10 \%$ & 9 \\
\hline 93 & Systems theory; control & 6 & 6 & $6.70 \%$ & 6 \\
\hline 92 & $\begin{array}{l}\text { Biology and other natural } \\
\text { sciences }\end{array}$ & 13 & 3 & $3.40 \%$ & 5 \\
\hline 37 & Dynamical systems & 20 & 2 & $2.20 \%$ & 4 \\
\hline 90 & $\begin{array}{l}\text { Operations research, } \\
\text { mathematical programming }\end{array}$ & 7 & 2 & $2.20 \%$ & 2 \\
\hline 11 & Number theory & 15 & 1 & $1.10 \%$ & 3 \\
\hline 33 & Special functions & 44 & 1 & $1.10 \%$ & 2 \\
\hline 40 & $\begin{array}{l}\text { Sequences, series, } \\
\text { summability }\end{array}$ & 53 & 1 & $1.10 \%$ & 2 \\
\hline 42 & Fourier analysis & 30 & 1 & $1.10 \%$ & 1 \\
\hline 46 & Functional analysis & 23 & 1 & $1.10 \%$ & 1 \\
\hline 49 & $\begin{array}{l}\text { Calculus of variations and } \\
\text { optimal control; optimization }\end{array}$ & 27 & 1 & $1.10 \%$ & 1 \\
\hline 60 & $\begin{array}{l}\text { Probability theory and } \\
\text { stochastic processes }\end{array}$ & 10 & 1 & $1.10 \%$ & 1 \\
\hline 26 & Real functions & 32 & 0 & $0.00 \%$ & 1 \\
\hline 30 & $\begin{array}{l}\text { Functions of a complex } \\
\text { variable }\end{array}$ & 26 & 0 & $0.00 \%$ & 1 \\
\hline 39 & $\begin{array}{l}\text { Difference and functional } \\
\text { equations }\end{array}$ & 35 & 0 & $0.00 \%$ & 1 \\
\hline 41 & $\begin{array}{l}\text { Approximations and } \\
\text { expansions }\end{array}$ & 42 & 0 & $0.00 \%$ & 1 \\
\hline 94 & $\begin{array}{l}\text { Information and } \\
\text { communications, circuits }\end{array}$ & 9 & 0 & $0.00 \%$ & 1 \\
\hline & Totals & & 89 & & \\
\hline
\end{tabular}

Table 1. The Rank in MRDB is the relative frequency of the 2-digit MSC subject area using Math Reviews data for articles published in journals from 2008 to 2018 (inclusive). The Count is the number of math HCRs for whom this is the most frequent subject in which they publish.The \% in HCR is the percentage of math HCRs for whom this is the most frequent subject in which they publish. The Count+ in HCR is the number of math HCRs for whom this is either the most frequent subject in which they publish, or a secondary subject for them. To be a secondary subject, the author should have at least half as many publications in the subject as in their most frequent subject. 


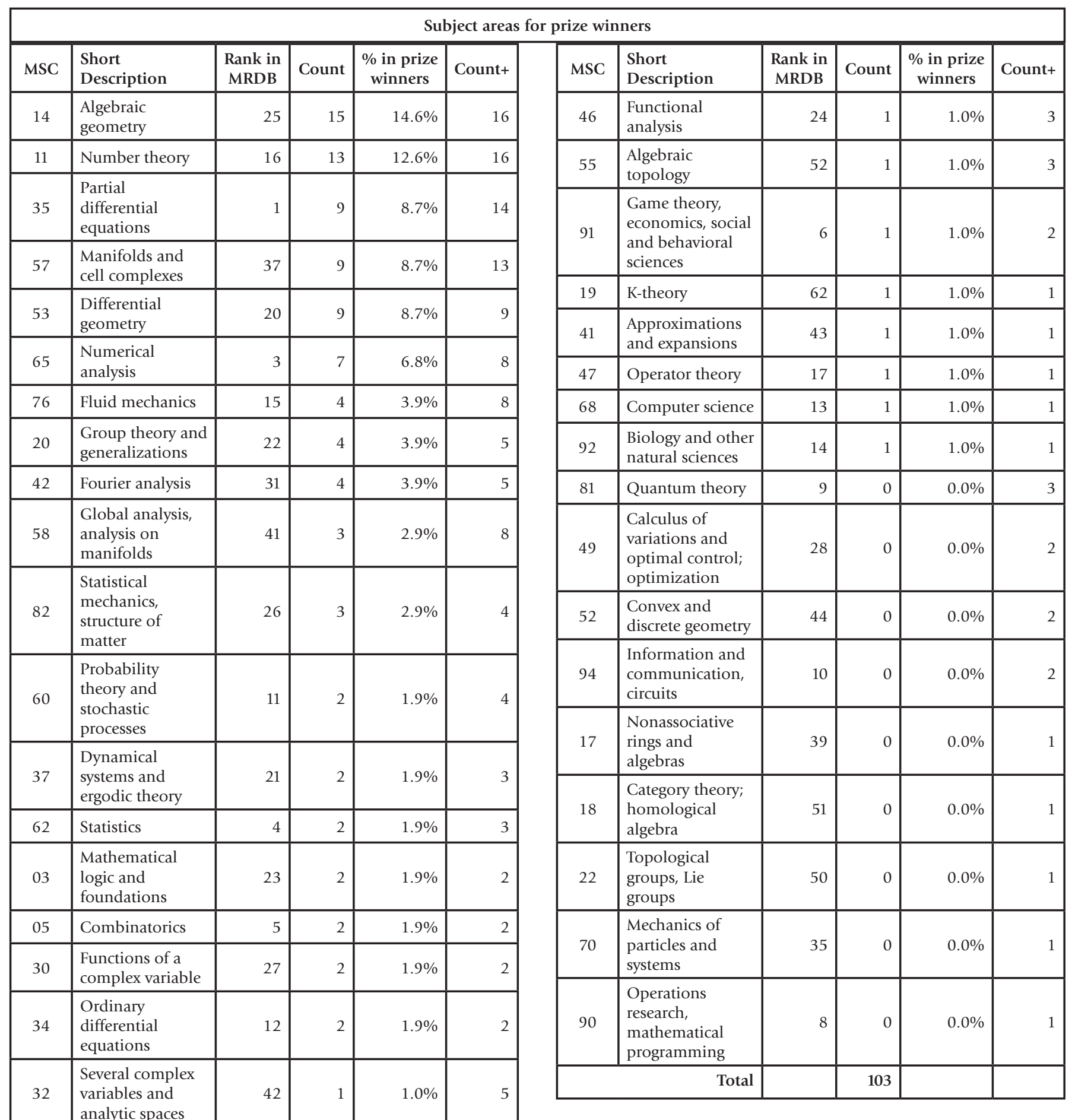

Table 2. The Rank in MRDB is the relative frequency of the 2-digit MSC subject area using Math Reviews data for articles published in journals from 2008 to 2018 (inclusive). The Count is the number of the prize winners who have won at least two society prizes and for whom this is the most frequent subject in which they publish. The $\%$ in prize winners is the percentage of the prize winners for whom this is the most frequent subject in which they publish. The Count+ in prize winners is the number of the prize winners for whom this is either the most frequent subject in which they publish, or a secondary subject for them. To be a secondary subject, the author should have at least half as many publications in the subject as in their most frequent subject. 
The two lists differ fundamentally in the way they have been created. Highly Cited Researchers are identified based on the counts of citations to their papers. This is, essentially, a one-dimensional process. Such a measure is likely to be a projection of many characteristics onto one axis. For instance, it is possible for a researcher to publish a few papers, each of which garners a lot of citations. It is also possible for a researcher to publish a lot of papers, each of which garners a few citations. Numerically, it is hard to say who is the more influential mathematician. Should we instead be counting citations normalized by number of papers, similar to the Impact Factor or the MCQ? Should long papers be weighted more than short papers?

The prize winners are identified by committees of active researchers who have been chosen by one of the professional societies to identify noteworthy researchers. The committees change membership from year to year. Committees from different societies are likely to draw from different pools, at least to some extent. Needless to say, prizes and awards have to contend with biases, both explicit and implicit.

\section{Self-Referencing/Self-Citing Comparisons Across Datasets}

This column was partially motivated by the paper [SPA] about self-citing and self-referencing in the sciences, using data from Clarivate Analytics (creators of Web of Science and Journal Citation Reports). Here, I use the definitions of those terms by Szomszor, Pendlebury, and Adams in [SPA]. The difference between the two terms is subtle, but meaningful. Self-referencing considers how often an author's citations (in their own papers) are to their own works, as compared to citing other authors. Self-citing considers how many citations to an author's works come from the author's own publications. For example, if your self-referencing score is 11.23 and your self-citing score is 3.57 , that means that $11.23 \%$ of the references in your papers are to your previous papers, and $3.57 \%$ of all the papers that cite your papers are papers that you authored. Szomszor, Pendlebury, and Adams study similarities and differences in self-citing and self-referencing across disciplines by examining the self-citing and self-referencing trends among HCRs by subject area. In setting up their study, they assert that the behavior of the cohort of HCRs "should serve as a bellwether for culturally appropriate citation practice." As pointed out already, it is not clear that the HCRs in mathematics are typical or exceptionally influential.

MathSciNet uses the data in the Mathematical Reviews database, which I abbreviate as the "MR database" or as "MRDB." The MR database is similar to the Clarivate database that underlies Web of Science and Journal Citation Reports, but with some significant differences. First of all, the MR database is limited to information from mathematical research publications, though that includes theoretical physics, mathematical biology, and other areas of applications. Second, the citations in the MR database are from a select subset of about 650 journals out of our total coverage of approximately 1800 journals. In our editorial decisions for coverage of journals, we try to exclude weak journals. In our decisions for counting citations, we look for strong journals. This serves to reduce opportunities for certain types of self-citation and self-referencing.

With the help of Erol Ozil, the IT manager at Mathematical Reviews, I queried the MR database to find the 1000 authors with the highest citation counts, using Mathematical Reviews data from journal articles from 2010 to 2020. Statistics on self-referencing and self-citing rates were computed for the whole set of 1000 top-cited authors and for the subset of 100 top-cited authors.

We also compared the statistics from [SPA] with the statistics for the prize winners. Using the list of prize winners, we matched the names with authenticated names in the database. We queried the MR database to look at the self-citing and self-referencing behaviors of the prize winners. Some prize winners did not occur in the database. Their citation counts and self-referencing/self-citing counts were left blank. Also, some of the prizes have been around for a long time, with some prizes having been awarded in the 1970s. Consequently, some of those prize recipients had no papers published in recent times, and their work fell outside the period for which we have citation information. Therefore, when computing statistics, we restricted the list of prize winners to those who had received the prize in the year 2000 through the present. Otherwise, a large number of zero values would have artificially decreased the statistics.

The first table shows the statistics for self-referencing for the 89 HCRs in mathematics, the top 100 and top 1000 cited researchers from the MR database, and the 365 mathematicians who had won at least one of the AMS, EMS, IMU, or SIAM prizes between 2000 and the present. The second table shows the statistics for self-citing for the same four cohorts. IQR is the interquartile range, defined as the difference between the third quartile (Q3) and the first quartile (Q1). Table 3 is analogous to Table 2 in [SPA] Table 4 is analogous to Table 3 in [SPA].

\begin{tabular}{lrrrrr}
\hline Cohort & \multicolumn{2}{c}{$\begin{array}{c}\text { Distribution } \\
\text { parameters }\end{array}$} & \multicolumn{2}{c}{ Indicative } \\
& Q1 & Median & Q3 & \multicolumn{2}{c}{$\begin{array}{c}\text { Lower, } \\
\text { Lowier thresholds }\end{array}$} \\
& & & & Upper, \\
& 11.51 IQR +3.0 IQR \\
\hline HCR & 15.63 & 22.47 & 38.47 & 54.47 \\
Top 100 cited & 6.93 & 9.08 & 12.01 & 19.62 & 27.24 \\
Top 1000 cited & 5.05 & 6.96 & 9.42 & 15.99 & 22.55 \\
Prize Winners & 5.68 & 7.91 & 11.26 & 19.63 & 29.99 \\
\hline
\end{tabular}

Table 3. Key parameters for rates of self-referencing percentages (citing to earlier papers). 


\begin{tabular}{|c|c|c|c|c|c|}
\hline \multirow[t]{2}{*}{ Cohort } & \multicolumn{3}{|c|}{$\begin{array}{l}\text { Distribution } \\
\text { parameters }\end{array}$} & \multicolumn{2}{|c|}{$\begin{array}{c}\text { Indicative } \\
\text { outlier thresholds }\end{array}$} \\
\hline & Q1 & Median & Q3 & $\begin{array}{l}\text { Lower, } \\
+1.5 \mathrm{IQR}\end{array}$ & $\begin{array}{l}\text { Upper, } \\
\text { 3.0 IQR }\end{array}$ \\
\hline HCR & 6.02 & 12.91 & 20.61 & 42.48 & 64.36 \\
\hline Top 100 cited & 3.77 & 5.51 & 7.54 & 13.20 & 18.86 \\
\hline Top 1000 cited & 4.94 & 6.87 & 9.79 & 17.06 & 24.33 \\
\hline Prize Winners & 0.00 & 0.22 & 2.42 & 6.06 & 9.69 \\
\hline
\end{tabular}

Table 4. Key parameters for rates of self-citing percentages (citing from later papers).

The differences in the statistics are dramatic. For self-referencing, the HCR median is 1.72 times the median of the top 100 cited authors in the MR Database and nearly twice the median of the prize winners. Similar ratios hold for the other statistics. For self-citing, the HCR median is 2.34 times the median of the top 100 cited authors in the MR Database and 58 times the median of the prize winners.

It is interesting to see the differences between self-referencing and self-citing using the Mathematical Reviews data. For instance, as the cohort grows from the top 100 to the top 1000, the amount of self-referencing goes down and the amount of self-citing goes up. I am also struck by the extremely low rate of self-citing among the prize winners.

Szomszor, Pendlebury, and Adams use their statistical analysis of the Clarivate data to draw conclusions from the data for HCRs about the sociology of science as specialized to mathematics. I see two reasons to question this. First, using the HCRs in mathematics as proxies for the entire cohort of mathematical researchers does not seem right. The HCRs are not representative by subject areas and were not selected by a random sampling process. Second, the comparison with other datasets indicates that drawing conclusions from the data for HCRs about the sociology of science as specialized to mathematics is not warranted. By definition, the HCR identifies people who are good at accumulating a large number of citations. There are multiple ways to achieve this, and when citations alone are the measure, they are all rewarded equally.

\section{References}

[SPA] M. Szomszor, D. A. Pendlebury, and J. Adams, How much is too much? The difference between research influence and self-citation excess, Scientometrics 123 (2020), 11191147. https://doi .org/10.1007/s11192-020-03417-5

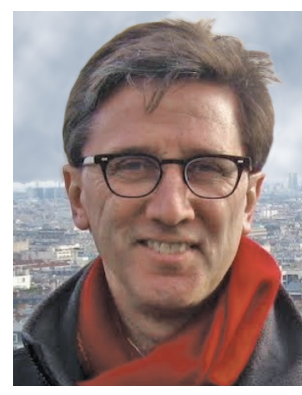

Edward Dunne

\section{Credits}

Author photo is courtesy of Edward Dunne.

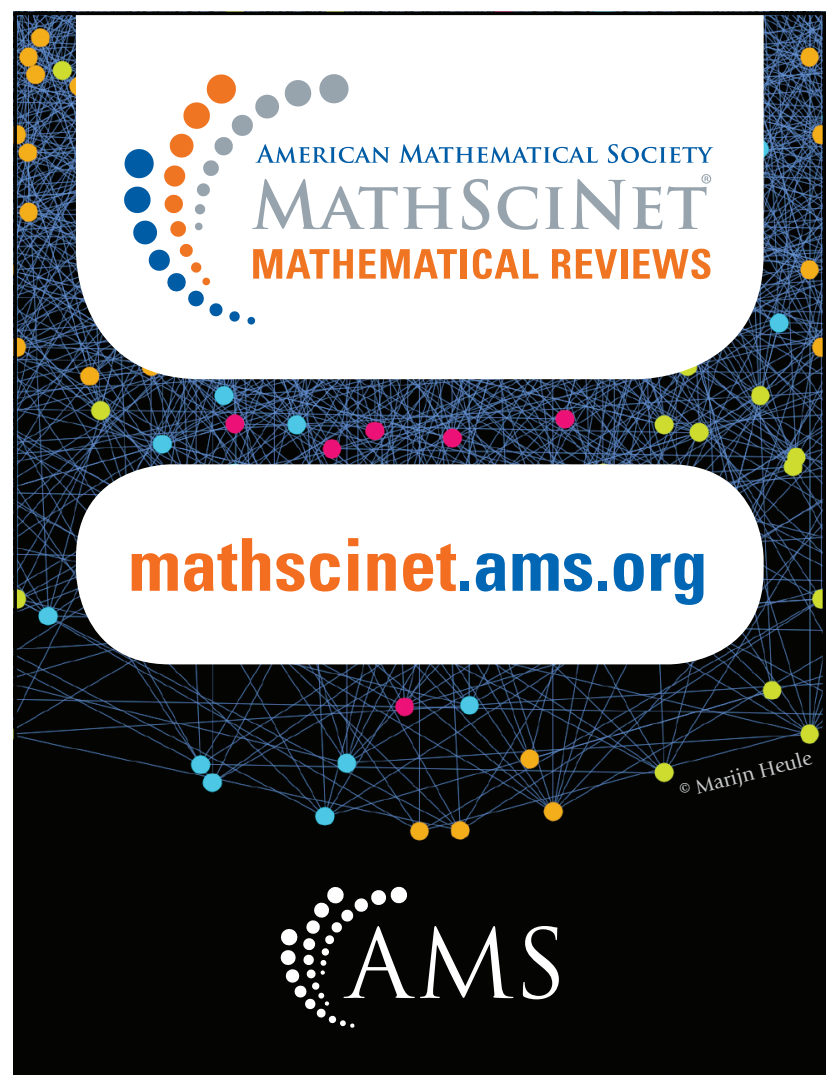

